\title{
Medical Aspects of Recruiting for the Brigade of Gurkhas
}

\author{
Lt Col G E Ratcliffe \\ $\mathrm{MB}, \mathrm{MRCP}, \mathrm{RAMC}$, Consultant Physician \\ Queen Elizabeth Military Hospital, Woolwich
}

\begin{abstract}
SUMMARY: The medical aspects of recruiting for the Brigade of Gurkhas are discussed with particular emphasis to the medical examination being the first sieve in the recruiting process. In 1987 there were 248 medical failures of a total of 913 recruits examined. The commonest causes of failure were an abnormal chest $X$-Ray and chronic middle ear disease with or without defective hearing.
\end{abstract}

\section{Introduction}

For over one hundred and fifty years Britain has recruited Gurkha soldiers. Until Indian independence in 1947 Gurkha regiments were part of the Indian Army, but since then the Brigade has been an integral part of the British Army with Gurkhas seeing active service in Malaya, Brunei, Borneo and the Falklands, as well as playing a major role in internal security in Hong Kong.

Recruiting depots were established in Nepal in the late 1950's and became operational in 1960. At present these depots are in Dharan in the East and Pokhara in the Centre West, although the closure of Dharan is imminent.

This paper examines the recruiting process with particular regard to the medical aspects. The recruiting process is a continual one. The Chief Recruiting Officer (CRO) is the Commander, British Gurkhas Nepal, and under his command are two deputies (DROs), one in Dharan, the other in Pokhara. Each DRO has several assistants (AROs), all of whom are retired Queens Gurkha Officers. They are responsible initially for hill selection of potential recruits. Each recruiting area, or Galla, produces a certain number of potential recruits under the jurisdiction of the Galla Wallah, a retired Gurkha NCO. Once chronological details are confirmed the AROs select a suitable number of these potential recruits at hill selection when the first very basic "medical" examination takes place. This includes height and weight measurements: the minimum height required is 62 inches $(157.5 \mathrm{~cm})$ and minimum weight is $110 \mathrm{lbs}(52 \mathrm{~kg})$. Candidates with obvious deformities or handicaps are immediately excluded. Simple educational tests take place also. The potential recruits selected are instructed to appear at their respective depot for assessment at a date in the near future.

Shortly after arrival in either Dharan or Pokhara, once initial documentation procedures are completed, a full medical examination by RAMC medical officers takes place.

The medical team consists of the consultant physician, consultant surgeon and consultant anaesthetist of BMH Dharan plus the visiting doctors from Hong Kong, usually two GDMOs plus the consultant radiologist from BMH Hong Kong.

The medical examination is the first sieve to reduce the potential number of recruits from whom the final selection will be made. Strict adherence to the Pulheems pamphlets ${ }^{1}$ is followed and in each case of failure, the reason is explained with particular emphasis being paid to possible future attempts at recruitment. For example, a presumptive ventricular septal defect this year will fail again next year, whereas mild to moderate acne vulgaris this year may not preclude possible acceptance indefinitely.

The standard documentation (F Med 1) is used but it is important to emphasise that the previous medical history section may well be inaccurate in its information. Medical services are poorly developed in Nepal and medical documents virtually non-existent. Despite careful questioning by locally employed medical staff, errors can and do occur: for example a recurrence of Grand Mal seizures can occur during basic training. Fortunately such occurrences are very rare but there is no way to avoid them without extending the basic examination to unacceptable levels of expense. Great emphasis is paid to the clinical examination therefore and any possible query generally results in medical nonacceptance. Competition is fierce and the importance of the medical examination is appreciated by the potential recruits and success or failure can be consequently accepted with sheer delight or abject misery.

\section{Results}

For recruitment in January 1987, 3026 had attended hill selection in the West and 3264 in the East. Of these, 477 potential recruits were selected in the West and 436 in the East. Thus 1 in 7 were selected in the hills $(14.7 \%)$.

Of the 913 potential recruits examined, 256 were failed on medical grounds, a ratio of 1 in $3.6(28 \%)$ (Table 1).

A few further comments are necessary on some of the reasons for failure. Chest X-Rays were carefully reviewed by the visiting Consultant Radiologist. Many showed minor abnormality e.g. spots of calcification or a healed Ghon focus which were accepted. However, if there was evidence of reduced lung volume e.g. by a displaced horizontal fissure, or there was evidence of pulmonary fibrosis, an obviously raised hemidiaphragm, tracheal deviation, marked pleural and/or apical calcification, then such abnormalities resulted in 
failure. Cardiomegaly on X-Ray also resulted in failure. Obviously cases of active tuberculosis were rejected.

In some cases where doubt existed lateral and apical views were obtained before decisions were made. Dextrocardia without situs inversus was noted on one occasion. This potential recruit was accepted medically but he was rejected later in the recruitment process.

All recruits with diastolic and pansystolic murmurs were automatically rejected. Mid-systolic or ejection murmurs were assessed more carefully, the effects of exercise noted, and decisions taken. ECGs were not routinely performed.

A single raised blood pressure was noted and repeated on at least two more occasions during the day and was assessed as hypertension only if the result remained high.

Proteinuria was automatically a reason for failure if albustix showed one plus or more of protein. 24 hour urinary protein estimations were not performed.

Pes planus is common in Nepal: if foot mobility was normal the recruit was medically accepted. Other foot deformities were generally not accepted.

Some scarring of tympanic membranes was acceptable assuming audiograms were normal and Valsalva manoeuvre revealed normal movement. Mildly scarred eardrums are common in Nepal, and this problem was discussed with the Consultant Adviser in ENT during one of his routine visits (personal communication).

Clerks for the Brigade of Gurkhas require a lower standard of visual acuity and a few appeared wearing

Table 1: Causes of Recruit Medical Failure

\begin{tabular}{lr}
\hline Disability & Number \\
\hline Visual Acuity & 8 \\
Colour Blindness (CP4) & 5 \\
Chronic Middle Ear Disease with or without & \\
$\quad$ Defective Hearing & 39 \\
Abnormal CXR & 97 \\
Cardiac Murmur & 21 \\
Asthma/Bronchospasm & 5 \\
Hypertension & 9 \\
Skin diseases, mainly acne & 19 \\
Scoliosis & 3 \\
Cryptorchidism & 1 \\
Hydrocoele & 1 \\
Haematuria & 7 \\
Proteinuria & 19 \\
Pes Planus & 7 \\
Varicose Veins & 7 \\
Foot deformity, hallux valgus & 8 \\
\cline { 2 - 2 } & 256 \\
\hline
\end{tabular}

spectacles: unfortunately two were failed because their prescription did not alter their unaided visual acuity.

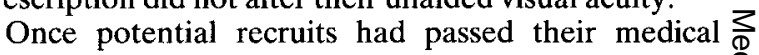
examinations they returned to the respective depot to $\varrho$ continue the recruiting process. This period lasted two $\delta$ to three weeks during which various physical and educational assessments took place: occasionally further medical opinion was sought if such problems as failed $\stackrel{\vec{\rho}}{\rightarrow}$ BFT occurred. Sometimes a medical reason was $\overrightarrow{0}$ discovered and a previous decision of medical success was reversed.

\section{Discussion}

Despite very careful assessment of the potential Gurkha recruit the decision to fail an individual remains difficult but necessary to maintain the high standards of $\overrightarrow{\vec{\omega}}$ the Brigade. Some may argue that the medical $\omega_{\sigma}$ examination should not be the first reason to reject a $\overrightarrow{\vec{D}}$ potential recruit. However, the recruiting process is complicated and an obvious medical abnormality which ? enforces rejection saves time and money, the failure $\vec{\omega}$ usually leaving the respective depot within a few days. Any failures with active tuberculosis, for example, were N offered treatment which was usually accepted. Although surgical correction of varicose veins, for example, wae 음 offered to the failed recruit in the general context of the $\vec{e}$ hospital's work, it was stressed that this would not guarantee recruitment in the future.

Of the 665 potential recruits originally examined whe were medically fit, 256 were accepted as recruits: thus of an original 6290 chosen to attend hill selection a mere $4 \%$ were finally recruited. This figure only confirms the intensity of competition.

With the future of the Brigade of Gurkhas assure $\bar{d}$, but with the imminent closure of BMH Dharan and hence no service medical officers permanently in Nepal, $\unrhd$ there will be a requirement for a specialist team to visit $\overrightarrow{\overrightarrow{0}}$ Pokhara annually for recruiting, with the first visiting team due in the Spring of 1990.

\section{Acknowledgement}

I am grateful to the visiting medical officers, my consultant colleagues in Dharan plus the local employed civilian medical staff of BMH Dharan for their assistance in this important work. Notwithstanding the many civilian patients seen in BMH Dharan, the recruiting process was one of the raisons d'etre for its existence in the first place.

\section{REFERENCE}

1. JSP 346 PulHeEMs. Systems of Medical Classification 1976. 